\title{
HandyRef-K: Comparison of the Latest Handheld Auto Refracto-keratometer with Retinomax and Plusoptix in Patients Younger than Three Years of Age
}

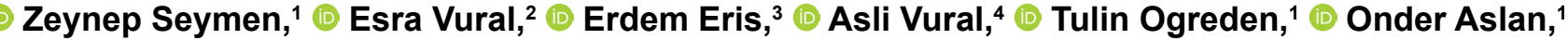 \\ (1) Ali Riza Cenk Celebi, ${ }^{5}$ (이 Irfan Perente ${ }^{3}$
}

1Department of Ophthalmology, University of Health Sciences Istanbul Suleymaniye Training and Research Hospital, Istanbul, Turkey ${ }^{2}$ Department of Ophthalmology, Mardin State Hospital, Mardin, Turkey

${ }^{3}$ University of Health Sciences Prof. Dr. N. Resat Belger Beyoglu Eye Training and Research Hospital, Istanbul, Turkey

${ }^{4}$ Department of Ophthalmology, Bakırkoy Sadi Konuk Training and Research Hospital, Istanbul, Turkey

${ }^{5}$ Department of Ophthalmology, Acıbadem University Faculty of Medicine, Istanbul, Turkey

\begin{abstract}
Objectives: The purpose of the study was to compare the refractive error measurements of pediatric patients performed with a Plusoptix A09 photorefractor (PO; Plusoptix AG, Nuremberg, Germany), a Retinomax K-plus 3 (RTX; Right Group, Tokyo, Japan), and the new handheld auto refracto-keratometer, the Nidek HandyRef-K (HDY; Nidek SA, Créteil, France), and to evaluate the intermethods agreement.

Methods: A total of 194 eyes of 194 children were included in the study. All of the children underwent refraction measurement with the PO before cycloplegia and 2 autorefractors were used after cycloplegia: the RTX and the HDY.

Results: The mean age of the patients was 16.65 10.04 months (range: 3-34 months). There were no statistically significant differences between the spherical values (SV) or cylindrical axis values (CAV) measured with the PO (SV: I.6I II.79 diopters [D]; CAV: $94.25 \pm 72.47 \mathrm{D})$, the RTX (SV: I.9I $\pm 2.06 \mathrm{D}$; CAV: $94.3 \pm 73.44 \mathrm{D}$ ), and the HDY (SV: I.89 $\pm 2.04 \mathrm{D}$; CAV: $93.55 \pm 73.7 \mathrm{I} D)(p>0.05)$. There was a statistically significant difference in the cylindrical values (CV) assessed with the RTX (CV: $-0.97 \pm 0.75 \mathrm{D})$ and the HDY $(C V:-1.1 \mathrm{I} \pm 0.76 \mathrm{D})(p=0.003)$ and the HDY and the PO $(C V:-0.92 \pm 0.68 \mathrm{D})$ $(p=0.002)$, while there was no statistically significant difference between the values determined with the RTX and the PO $(p>0.05)$. Statistically significant differences were demonstrated for spherical equivalent values (SEV) obtained with the RTX (SEV: I.43 $\pm 1.97 \mathrm{D})$ and the PO (SEV: I.I5 $\pm 1.74 \mathrm{D})(p=0.02)$, and the HDY (SEV: I.34 $\pm 1.95 \mathrm{D})$ and the PO ( $p=0.03)$, but no significant difference was found between the RTX and the HDY values ( $p>0.05)$.

Conclusion: No significant difference was found between the SEV measured by the RTX and the HDY, but the PO was significantly less hyperopic than the RTX and the HDY. The CV measured by HDY was higher than that of other devices. These devices can be used for screening in crowded pediatric ophthalmology clinics and may be an easier way of measuring refractive errors in children younger than 3 years of age, but high SEV and CV results should serve as an alert to physicians. It should also be kept in mind that cycloplegic retinoscopy is still the gold standard and these alternative methods can only be used for screening. The prescription of eyeglasses should not be made without cycloplegic retinoscopy.

Keywords: Amblyopia, HandyRef, Plusoptix, refractometer, Retinomax, retinoscopy.
\end{abstract}

\section{Introduction}

Early diagnosis and correction of refractive defects in children is very important for the prevention of amblyopia and secondary preventable visual loss (I). However, diagnostic controversies still exist among ophthalmologists on the issue of accurate diagnosis of significant refractive defects. The traditional method for diagnosing refractive defects in childhood is cycloplegic retinoscopy performed by an experienced ophthalmologist. However, this requires a long period of time and patient compliance, and this method can be difficult, even for

Address for correspondence: Zeynep Seymen, MD. Istanbul Suleymaniye Egitim ve Arastirma Hastanesi, Saglik Bilimleri Universitesi, Oftalmoloji Anabilim Dali, Istanbul, Turkey

Phone: +90 2124986161 E-mail: dr.zeynepseymen@hotmail.com

Submitted Date: August 27, 2018 Accepted Date: December 28, 2018 Available Online Date: February 26, 2019

${ }^{\circ}$ Copyright 2019 by Beyoglu Eye Training and Research Hospital - Available online at www.beyoglueye.com 
experienced ophthalmologists $(2,3)$. Therefore, efforts are being exerted to develop alternative tools to retinoscopy that require minimal patient cooperation and allow for faster and easier evaluation in clinics with a high workload.

Many such refractometers with rapidly improving design and greater claims to exactness are available (I). Photoscreening is useful, as it requires less time than the traditional method and permits faster and easier evaluation in clinical practice $(4,5)$. Hand-held refractors, such as the HandyRef-K (HDY; Nidek SA, Créteil, France) and the Retinomax K-plus 3 (RTX; Right Group, Tokyo, Japan), have become more important in recent years because of the busy clinical schedule of ophthalmologists, increasing confidence of patients in sophisticated mechanical devices, and the capability to detect refractive errors rapidly $(I, 2)$. Several authors have already studied the accuracy and reproducibility of RTX as a screening device (6-8). HDY is the latest handheld auto refracto-keratometer.

Each refractometer has its own advantages. Both of these handheld auto refracto-keratometers have the advantage of measuring refractive errors in the supine position and measuring keratometric values. The PO photorefractor has the ability to measure the pupil size of both eyes simultaneously before cycloplegia. This gives it an advantage over the other 2 methods in detecting anisocoria $(9,10)$.

The aim of the present study was to compare refractive error measurements of pediatric patients performed with the PO photorefractor, the RTX, and the HDY handheld auto refracto-keratometer in outpatient clinic conditions and to evaluate the intermethods agreement. To the best of our knowledge, this is the first and only study comparing the latest Nidek auto refracto-keratometer with 2 other refraction methods in patients younger than 3 years of age.

\section{Methods}

A total of 194 consecutive patients were evaluated in a routine ophthalmological assessment. Participants were recruited from Süleymaniye Obstetrics and Pediatrics Training and Research Hospital between July I, 2016, and January 3I, 2017. Written informed consent was obtained from all of the parents prior to the procedure. The study was approved by the medical Ethics Committee of Bakırkoy Sadi Konuk Training and Research Hospital and was performed in accordance with the Declaration of Helsinki.

Demographic data including sex, date of birth, age at examination, and any ocular or systemic diseases were recorded. Children with mental retardation, presence of systemic disease, strabismus, nistagmus, previous ocular surgery, or trauma were excluded from the study. Patients younger than 36 months of age without any anterior or posterior segment pathologies other than refractive error were included in the study. Patients older than 36 months were excluded.
A comprehensive ophthalmic examination, including an orthoptic examination, cover-uncover test, strabismus and nistagmus evaluation, and anterior and posterior segment examination using handheld biomicroscopy and binocular indirect ophthalmoscopy was performed on each patient. None of the measurements were performed under general anesthesia.

All of the children underwent refraction measurement with the PO photorefractor, followed by cycloplegic drop administration and assessment with 2 different autorefractors, the RTX and the HDY. The measurements were performed when no pupillary activity was observed.

The measurements taken with the PO were performed under darkroom conditions at a distance of $I$ meter in front of the patient while visualizing both eyes binocularly from the monitor. The measurement range for this device is -7.0 diopters (D) to +5.0 D for spherical and cylindrical values. If the SEV is out of range, the measurement value only displays "hyperopia" or "myopia." Ocular misalignment $\geq 10^{\circ}$ could not be measured binocularly and was adjusted to a sequential monocular measurement mode. The minimum pupil size required for the measurement was $4.0 \mathrm{~mm}$; the maximum pupil size was $8.0 \mathrm{~mm}$.

The RTX auto refracto-keratometer was used at a distance of about $50 \mathrm{~mm}$ from the patient. The RTX device is also a keratometer, and can measure vergence, pupil size, and accommodation simultaneously. It has a reported measurement range of -18.0 to $+23.0 \mathrm{D}$ for spheres and 0 to $\pm 12.0 \mathrm{D}$ for cylinders. The minimum pupil size required for measurement is $2.3 \mathrm{~mm}$. The RTX also has a retro-illumination mode.

The HDY auto refracto-keratometer, which is a handheld device, was used at a distance of about $46 \mathrm{~mm}$ from the patient. The HDY is also a keratometer and can measure the pupil size. It has a measurement range of -20.0 to +20.0 $\mathrm{D}$ for spheres and 0 to $\pm 12.0 \mathrm{D}$ for cylinders. The minimum pupil size required for measurement is $2.0 \mathrm{~mm}$. The device has the ability to measure refraction with a small pupil size. The HDY also has a retro-illumination mode.

Spherical equivalent (SE), sphere power plus half of the cylinder power), spherical power (SP), cylindrical power (CP), and cylindrical axis (CA) measurements were recorded. Astigmatism was recorded in minus cylinder notations. The following formula was used for the calculation of SE: $\operatorname{SE}(D)=-$ sphere(D)+[cylinder (D)/2].

All statistical analyses were performed using SPSS for Windows, Version 15.0 (SPSS Inc., Chicago, IL, USA). The results were reported as the mean $\pm S D$, frequency, and percentage. Differences between the measurements were tested for significance by repeated measures analysis of variance with Bonferroni-adjusted pairwise comparisons. A P value of $<0.05$ was considered statistically significant. The Pearson correlation coefficient was used to evaluate the association between the measurements taken with the RTX, 
HDY, and PO. A p value $<0.01$ was considered statistically significant. Bland-Altman plots were used to examine the agreement between methods.

\section{Results}

A total of 194 right eyes of 194 children were included in the study. RTX and HDY measurements could be performed in all 194 patients. PO measurements could be obtained from only 102 eyes of 102 patients; therefore, 92 eyes were excluded when performing the statistical analysis to compare the RTX and HDY with the PO. PO measurements could not be obtained from patients with a pupil size smaller than $4 \mathrm{~mm}$ or wider than $8 \mathrm{~mm}$, with a refractive error measurement outside the range for spherical values (SV) and cylindrical values $(\mathrm{CV})$ of $-7.0 \mathrm{D}$ to $+5.0 \mathrm{D}$, and patients who were unable to fixate on the target. In this study, PO measurements were successfully obtained from 8 infants younger than 6 months of age ( 6 participants were 3 months old, 2 were 4 months old).

The mean age of the patients was $16.65 \pm 10.04$ months (range: 3 to 34 months). In this study group, 47 of the patients $(46.1 \%)$ were female and 55 of the patients $(53.9 \%)$ were male.
The mean $( \pm S D)$ SV, CV, CA and SE power measurements using the PO, RTX, and HDY are summarized in Table I.

There were no statistically significant differences in spherical or cylindrical axis measurements between the 3 devices $(p>0.05)$.

Analysis of variance (ANOVA) testing for CV indicated a statistically significant difference between the 3 devices $(p<0.00 I ; F=9.66)$. When measurements of the 3 devices were compared as binary groups, there was a statistically significant difference between the RTX and the HDY, and the HDY and the PO, while there was no statistically significant difference between the RTX and the PO $(p=0.003, p=0.002$, $p>0.05$, respectively).

Spherical equivalent values (SEV) obtained with PO were significantly less hyperopic than those with RTX and HDY, and there was a statistically significant difference between groups $(p=0.023)$. When binary groups were compared, statistically significant differences were demonstrated between the RTX and PO, and HDY and PO $(p=0.02, p=0.03$, respectively) but no significant difference was found between the results of the RTX and HDY ( $>00.05)$. The ANOVA testing results are summarized in Table 2 .

Table I. The mean $( \pm S D)$ spherical power, cylindrical power, cylindrical axis, and spherical equivalent power values as measured using the Plusoptix A09, Retinomax K-Plus 3 and the HandyRef-K handheld auto refracto-keratometer

\begin{tabular}{|c|c|c|c|c|}
\hline & Plusoptix A09 & Retinomax K-Plus 3 & HandyRef-K & $\mathbf{p}^{*}$ \\
\hline Spherical power (D) & $1.61 \pm 1.79(-2.25-+6.0)$ & $1.91 \pm 2.06(-2.25-+7.25)$ & $1.89 \pm 2.04(-2.25-+7.50)$ & $p>0.05$ \\
\hline \multicolumn{5}{|l|}{$\operatorname{mean} \pm S D(\min$ to $\max )$} \\
\hline Cylindrical power (D) & $-0.92 \pm 0.68(-3.50-0)$ & $-0.97 \pm 0.75(-3.0-0)$ & $-I .1 I \pm 0.76(-3.0-0)$ & $p<0.001$ \\
\hline \multicolumn{5}{|l|}{ mean $\pm S D(\min$ to $\max )$} \\
\hline Axis value (D) & $94.25 \pm 72.47(1-180)$ & $94.3 \pm 73.44(1-180)$ & $93.55 \pm 73.71(1-180)$ & $p>0.05$ \\
\hline \multicolumn{5}{|l|}{ mean $\pm S D(\min$ to $\max )$} \\
\hline Spherical equivalent (D) & 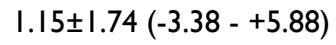 & $1.43 \pm 1.97(-2.88-+6.5)$ & $1.34 \pm 1.95(-3.0-+6.63)$ & $p=0.023$ \\
\hline $\operatorname{mean} \pm S D(\min$ to $\max )$ & & & & \\
\hline
\end{tabular}

*Analysis of variance testing; D: diopters; HandyRef-K: Nidek SA, Créteil, France; Plusoptix A09: Plusoptix AG, Nuremberg, Germany; Retinomax K-plus 3: Right Group, Tokyo, Japan.

Table 2. Comparisons of spherical, cylindrical, axis and spherical equivalent measurements between the HandyRef-K and the Retinomax K-Plus 3, the HandyRef-K and the Plusoptix A09, and the Retinomax K-Plus 3 and the Plusoptix A09

\section{HandyRef-Retinomax \\ Retinomax-Plusoptix \\ HandyRef-Plusoptix}

$$
p>0.05
$$

$\mathrm{p}=0.003$

$p>0.05$

$p>0.05$

$p>0.05$
$p>0.05$
$p>0.05$
$p=0.021$

$p>0.05$

$\mathrm{p}=0.002$

$p>0.05$

$\mathrm{P}=0.03$
ANOVA test between

the 3 devices

$$
\begin{gathered}
p>0.05 \\
p<0.001 \\
p>0.05 \\
p=0.023
\end{gathered}
$$

*Repeated measures analysis of variance (ANOVA) with Bonferroni-adjusted pairwise comparisons; HandyRef-K: Nidek SA, Créteil, France; Plusoptix A09: Plusoptix AG, Nuremberg, Germany; Retinomax K-plus 3: Right Group, Tokyo, Japan. 
According to the SEV, I 4 children (I3.7 \%) were myopic and 87 (85.2\%) were hyperopic in the PO group. In the HDY group, 16 children (15.6\%) were myopic and $83(81.3 \%)$ were hyperopic, while 15 children (I4.7\%) were myopic and 87 (85.2\%) were hyperopic in the RTX group.

The $95 \%$ limits of agreement for the SP, CP, axis, and SE measurements between the 3 devices are provided in Table 3 .

Bland-Altman analysis revealed good agreement between the HDY and RTX on SV and axis measurements, but there was no agreement on CV and SEV. Good agreement was found between the HDY and the PO on SEV and axis, but there was no agreement on CV and SV. Good agreement was found between the RTX and the PO on CV and axis, but there was no agreement on SEV and SV. There was no agreement between the HDY and the other 2 devices (RTX and $P O)$ on $C V$ measurements ( $=0.00 \mathrm{I}, \mathrm{p}=0.00 \mathrm{I}$, respectively).

There was a significant positive correlation for SV, CV, CA, and SEV between the HDY and RTX, HDY and PO, and the RTX and PO [( $r=0.979, r=0.858, r=0.898, r=0.976)$; $(r=0.728, r=0.738, r=0.764, r=0.728) ;(r=0.733, r=0.684$, $r=0.856, r=0.744)$, respectively; $(p=0.000 I)]$.

\section{Discussion}

Evaluating the accurate measurement of refractive errors in children younger than 36 months of age is still a challenge in routine clinical practice, even for an experienced pediatric ophthalmologist. Assessment of refractive errors requires certain capabilities from the instrument and some degree of experience from the user. The most accurate measurements can be taken when there is good cooperation; therefore, the device must be able to provide quick, accurate readings before a child loses the desire or ability to cooperate.

Cycloplegic retinoscopy is the gold standard method of measuring refractive errors, but it can require time and effort from the examiner owing to the potential lack of cooperation from a child and it is user dependent (I). Faster and accurate devices for measuring refractive errors are needed, particularly for very busy pediatric ophthalmology clinics.

Some devices were developed as an alternative to cycloplegic retinoscopy, such as photorefractometers (Plusoptix series), hand-held autorefractometers (Righton series) and tabletop autorefractors (Canon RK-FI, Nikon NRK 8000, etc.). The accuracy of these devices has been well established in studies (II-13). Yllmaz et al. (I4) compared the PO, RTX, and cycloplegic retinoscopy, and found good agreement between the 3 methods. They concluded that both of the devices can also be used as screening tools for children.

The Nidek HDY handheld auto refracto-keratometer is the latest handheld auto refracto-keratometer. This study compared the HDY with the RTX and the PO and evaluated the intermethods agreement. To the best of our knowledge, this is the first and the only study comparing the latest auto refracto-keratometer, the HDY, with 2 other refraction methods in patients younger than 3 years of age.

Table 3. Summary of variance between the HandyRef-K-Retinomax K-Plus 3 measurements, the HandyRef-K-Plusoptix A09 measurements, and the Retinomax K-Plus 3 and Plusoptix A09 measurements

\begin{tabular}{lcccc} 
& Mean difference & Standard deviation & 95\% limits of agreement & $\mathbf{P}$ \\
\hline HandyRef-K-Retinomax K-Plus 3 & & & & \\
SP & 0.01 & 0.42 & -0.06 to 0.10 & 0.642 \\
CP & -0.13 & 0.40 & 0.05 to 0.21 & 0.001 \\
Axis & -0.75 & 33.25 & -6.46 to 7.96 & 0.837 \\
SE & -0.08 & 0.43 & 0.00 to 0.17 & 0.042 \\
HandyRef-K and Plusoptix A09 & & & \\
SP & 0.28 & 1.43 & 0.00 to 0.56 & 0.048 \\
CP & -0.18 & 0.53 & -0.29 to 0.08 & 0.001 \\
Axis & -0.69 & 50.21 & -0.69 to -11.58 & 0.900 \\
O.19 & 1.37 & -0.08 to 0.46 & 0.167 & 0.19 \\
Retinomax K-Plus 3 and Plusoptix A09 & & & & \\
SP & 0.30 & 1.43 & 0.02 to 0.58 & 0.034 \\
CP & -0.05 & 0.57 & -0.16 to 0.06 & 0.370 \\
Axis & 0.05 & 39.22 & -8.45 to 8.57 & 0.989 \\
SE & 0.27 & 1.34 & 0.01 to 0.54 & 0.040 \\
\hline
\end{tabular}

*Bland-Altman analysis; CP: cylindrical power; SE: spherical equivalent; SP: spherical power; HandyRef-K: Nidek SA, Créteil, France; Plusoptix A09: Plusoptix AG, Nuremberg, Germany; Retinomax K-plus 3: Right Group, Tokyo, Japan. 
Schimitzek and Lagrèze (I5) reported that without cycloplegia, especially in children, the SEV obtained using a photorefractometer tended to be underestimated as a result of uncontrolled accommodation. However, while cycloplegia improves the accuracy of the SEV measurement, cycloplegia decreases the accuracy of CV and CA findings. In our study, we found that the results of SEV measurement using a photorefractor without cycloplegia were less hyperopic than those of other devices. These results support those of previous studies. In contrast, the CV obtained using the $\mathrm{PO}$ was lower than that of the other 2 methods. The CA and SP findings measured by the 3 devices were not statistically different.

Ozdemir et al. (16) compared noncyloplegic photorefraction with cycloplegic refraction measured with the PO device and cycloplegic retinoscopy or an autorefractometer. They could not obtain measurements from $10.9 \%$ of the participating children with the PO after cycloplegia due to enlarged pupil size after the administration of $1 \%$ cyclopentolate. They reported that the SE and the SV measured with cycloplegic photorefraction were statistically higher than the measurements of the other methods $(p<0.05)(16)$. The CV measured using cycloplegic refraction was statistically lower than the measurement achieved using photorefraction methods $(p<0.05)$; however, there was no significant difference in CA between the 3 methods ( $p>0.05$ ) (16). In our study, we performed PO photorefractor assessment without cycloplegia. The CV obtained with the HDY autorefractometer with cycloplegia was statistically higher than the noncycloplegic photorefraction method (PO). This may have been a result of the children squinting and the examiner's limited experience with this new device.

El-Defrawy et al. (17) demonstrated that measurements using RTX and retinoscopy with cycloplegia were similar for SV. Although the mean CV measured with the 2 methods was statistically different, this difference was clinically insignificant (0.23 D). Similarly, in our study, the mean CV measured using the RTX and HDY devices was statistically significant, but the difference was not clinically significant $(-0$. I3 D) $(p=0.003)$. There were no statistically significant differences in SV between the RTX and HDY ( $p>0.05)$.

Prabakaran et al. (18) reported that the mean SEV obtained with the RTX and cycloplegia was significantly less than the retinoscopy result (I.09 D) and astigmatism findings obtained with the RTX were significantly greater $(-0.89 \mathrm{D})$ than those of retinoscopy. The study sample consisted of children aged between 24 and 72 months (mean age: 52.3 months). Our study consisted of children aged between 3 and 34 months (mean age: 16.65 \pm 10.04 months). In our study, astigmatism measured with the HDY was higher than other measurement methods and the mean SEV measured by the PO device was less hyperopic than that of the other
2 devices. No significant difference was found between the RTX and the HDY in the assessment of SEV ( $p>0.05)$.

In the literature, most studies have evaluated the accuracy of refractometers in patients older than 3 years. Fewer comparative studies have been performed in the pediatric age group of those younger than 3 years (4). Peng et al. (19) compared cycloplegic refraction using a Retinomax device and cycloplegic retinoscopy in children aged between 5 months and 17 years of age and concluded that in children younger than 18 years of age, there was no clinical difference in the cycloplegic refraction determined by the Retinomax autorefractor when a pediatric ophthalmologist compared the results to those of retinoscopy. In our study, we evaluated children in the very challenging age group of those younger than 3 years. We agree that cycloplegic retinoscopy is still the gold standard for measuring refraction in pediatric patients, but in routine clinical practice we need faster yet still reliably accurate devices as alternatives to retinoscopy in order to achieve the needed results with young patients who have a short attention span.

In patients younger than 6 months, it is more difficult to obtain measurements by photorefractometer. In our study, PO measurements were successfully obtained from only 8 infants $(4.1 \%)$ younger than 6 months of age $(6$ were 3 months old, 2 were 4 months old). In order to get a measurement with the PO, the patient must be able to fixate on the target. It is very difficult for an infant to fixate on a distant target. Handheld autorefractometers may be a preferred choice for measuring refractive errors in such cases.

The primary limitation of our study is that we did not compare measurements between the 3 devices and cycloplegic retinoscopy. We performed cycloplegic retinoscopy in a very limited group of patients when the children became too restless and the families did not wish to continue to perform 3 different methods of refraction. This was a result of the very young and challenging age group we chose for our study.

The accuracy of the PO and RTX devices has been well established. There are many studies comparing these instruments with cycloplegic retinoscopy (II-13). We chose to compare these with the latest device. Furthermore, we aimed to evaluate the devices as an alternative to retinoscopy for measuring refractive errors in children younger than 3 years old. Further studies are needed to do a single comparison of HDY and cycloplegic retinoscopy.

The HDY handheld auto refracto-keratometer was determined to be a rapid and easy-to-use screening method of refraction, especially for ophthalmologists unskilled in retinoscopy. No significant difference in SEV was found between the RTX and the HDY. The CV measured using the HDY was higher than the results of the other devices; however, these differences were clinically insignificant. Although 
the SEV measured using the PO was significantly less hyperopic than that of the RTX and the HDY, photorefractometers can be a choice for rapid screening for amblyopia in children with atopia or cases in which the parents refuse cycloplegic eye drops. In routine clinical practice, these devices can be used in crowded pediatric ophthalmology clinics as a means of rapid screening, and may be the choice and an easier means of measuring refractive errors in children younger than 3 years of age, but a high SEV and CV result should alert the physician to consider the need for manual cycloplegic retinoscopy. Cycloplegic retinoscopy is still the gold standard for diagnosis, and these alternative methods can only be used for screening. Eyeglasses should not be prescribed without cycloplegic retinoscopy.

\section{Disclosures}

Ethics Committee Approval: This study was approved by the medical ethics committee of Bakırkoy Dr. Sadi Konuk Training and Research Hospital (number: 2017/169) on July 17, 2017.

Peer-review: Externally peer-reviewed.

Conflict of Interest: None declared.

Authorship Contributions: Involved in design and conduct of the study (ZS, EE, AV, TO, OA, IP); preparation and review of the study (ZS, EV, ARCC); data collection (ZS, EE, TO, OA); and statistical analysis (EE).

\section{References}

I. Rotsos T, Grigoriou D, Kokkolaki A, Manios N. A comparison of manifest refractions, cycloplegic refractions and retinoscopy on the RMA-3000 autorefractometer in children aged 3 to 15 years. Clin Ophthalmol 2009;3:429-3I. [CrossRef]

2. Williams C, Lumb R, Harvey I, Sparrow JM. Screening for refractive errors with the Topcon PR2000 Pediatric Refractometer. Invest Ophthalmol Vis Sci 2000;4I:103I-7.

3. Akil H, Keskin S, Çavdarli C. Comparison of the refractive measurements with hand-held autorefractometer, table-mounted autorefractometer and cycloplegic retinoscopy in children. Korean J Ophthalmol 2015;29: I 78-84. [CrossRef]

4. Moghaddam AA, Kargozar A, Zarei-Ghanavati M, Najjaran M, Nozari V, Shakeri MT. Screening for amblyopia risk factors in preverbal children using the Plusoptix photoscreener: a cross-sectional population-based study. Br J Ophthalmol 20I2;96:83-6.

5. Ruben J. Reimbursements and resources for pediatric vision screening. Am Orthop J 2006;56:54-6I. [CrossRef]

6. Liang $\mathrm{CL}$, Hung KS, Park N, Chan P, Juo SH. Comparison of measurements of refractive errors between the hand-held Retinomax and on-table autorefractors in cyclopleged and non- cyclopleged children. Am J Ophthalmol 2003; I36: I I 20-8.

7. Oral Y, Gunaydin N, Ozgur O, Arsan AK, Oskan S. A comparison of different autorefractors with retinoscopy in children. J Pediatr Ophthalmol Strabismus 2012;49:370-7. [CrossRef]

8. Ying GS, Maguire M, Quinn G, Kulp MT, Cyert L; Vision In Preschoolers (VIP) Study Group. ROC analysis of the accuracy of Noncycloplegic retinoscopy, Retinomax Autorefractor, and SureSight Vision Screener for preschool vision screening. Invest Ophthalmol Vis Sci 20I I;52:9658-64. [CrossRef]

9. Rajavi Z, Parsafar H, Ramezani A, Yaseri M. Is noncycloplegic photorefraction applicable for screening refractive amblyopia risk factors? J Ophthalmic Vis Res 2012;7:3-9.

10. Huang D, Zhang X, Wang Y, Zhu H, Ding H, Bai J, et al. Pupillary measurements and anisocoria in Chinese preschoolers 3-4 years of age screened using the plusoptiX AI2C. J AAPOS 2017;21:262.el-262.e5. [CrossRef]

I I. Payerols A, Eliaou C, Trezeguet V, Villain M, Daien V. Accuracy of PlusOptix A09 distance refraction in pediatric myopia and hyperopia. BMC Ophthalmol 2016;16:72. [CrossRef]

12. Harvey EM, Miller JM, Wagner LK, Dobson V. Reproducibility and accuracy of measurements with a hand held autorefractor in children. Br J Ophthalmol 1997;8I:94I-8. [CrossRef]

13. Farook M, Venkatramani J, Gazzard G, Cheng A, Tan D, Saw SM. Comparisons of the handheld autorefractor, table-mounted autorefractor, and subjective refraction in Singapore adults. Optom Vis Sci 2005;82:1066-70. [CrossRef]

14. Yilmaz I, Ozkaya A, Alkin Z, Ozbengi S, Yazici AT, Demirok A. Comparison of the Plusoptix A09 and Retinomax K-Plus 3 with retinoscopy in children. J Pediatr Ophthalmol Strabismus 20I5;52:37-42. [CrossRef]

15. Schimitzek T, Lagrèze WA. Accuracy of a new photo-refractometer in young and adult patients. Graefes Arch Clin Exp Ophthalmol 2005;243:637-45. [CrossRef]

16. Ozdemir O, Özen Tunay Z, Petriçli IS, Ergintürk Acar D, Erol MK. Comparison of non-cycloplegic photorefraction, cycloplegic photorefraction and cycloplegic retinoscopy in children. Int J Ophthalmol 20I5;8:128-3।.

17. el-Defrawy S, Clarke WN, Belec F, Pham B. Evaluation of a hand-held autorefractor in children younger than 6. J Pediatr Ophthalmol Strabismus 1998;35:107-9.

18. Prabakaran S, Dirani M, Chia A, Gazzard G, Fan Q, Leo SW, et al. Cycloplegic refraction in preschool children: comparisons between the hand-held autorefractor, table-mounted autorefractor and retinoscopy. Ophthalmic Physiol Opt 2009;29:422-6. [CrossRef]

19. Peng MY, Matta N, Silbert D, Tian J, Singman E. Accuracy of the Retinomax K-plus3 in measuring refractive error in a pediatric population. J AAPOS 20|4; |8:327-3I. [CrossRef] 